\title{
Extended phase space thermodynamics for black holes in a cavity
}

\author{
Peng Wang, Houwen Wu, Haitang Yang and Feiyu Yao \\ Center for Theoretical Physics, College of Physics, Sichuan University, \\ Chengdu, 610064, China \\ E-mail: pengw@scu.edu.cn, iverwu@scu.edu.cn, hyanga@scu.edu.cn, \\ yaofeiyu@stu.scu.edu.cn
}

ABSTRACT: In this paper, we extend the phase space of black holes enclosed by a spherical cavity of radius $r_{B}$ to include $V \equiv 4 \pi r_{B}^{3} / 3$ as a thermodynamic volume. The thermodynamic behavior of Schwarzschild and Reissner-Nordstrom (RN) black holes is then investigated in the extended phase space. In a canonical ensemble at constant pressure, we find that the aforementioned thermodynamic behavior is remarkably similar to that of the anti-de Sitter (AdS) counterparts with the cosmological constant being interpreted as a pressure. Specifically, a first-order Hawking-Page-like phase transition occurs for a Schwarzschild black hole in a cavity. The phase structure of a RN black hole in a cavity shows a strong resemblance to that of the van der Waals fluid. We also display that the Smarr relation has the same expression in both AdS and cavity cases. Our results may provide a new perspective for the extended thermodynamics of AdS black holes by analogy with black holes in a cavity.

KeYwords: Black Holes, Classical Theories of Gravity

ArXiv EPrint: 2006.14349v2 


\section{Contents}

1 Introduction 1

2 Phase structure of a Schwarzschild black hole in a cavity 3

$\begin{array}{lll}3 & \text { Phase structure of a RN black hole in a cavity } & 6\end{array}$

$\begin{array}{lll}4 & \text { Smarr relation } & 11\end{array}$

5 Discussion and conclusion $\quad 12$

\section{Introduction}

The area theorem of black holes [1] asserts that the total horizon area of black holes is a nondecreasing function of time in reasonable physical processes, which hints that black holes are endowed with thermodynamic properties. Since the area law bears a close resemblance to the second law of thermodynamics, Bekenstein postulated that black hole entropy is proportional to the horizon area $[2,3]$. The analogy between usual thermodynamics and black hole thermodynamics was further confirmed by the discovery of Hawking radiation, assigning black holes a temperature $[4,5]$. Analogous to the laws of thermodynamics, the four laws of black hole mechanics were established in [6].

Partly due to AdS/CFT correspondence [7], nearly fifty years after the discovery of black hole thermodynamics, understanding thermodynamic properties of various black holes, especially phase transitions of AdS black holes, is still a rather hot topic in the literature. Since AdS boundary plays a role of a reflecting wall, AdS black holes can be thermally stable in some cases, which makes it possible to study their phase behavior. In the seminal work [8], the Hawking-Page phase transition (i.e., a phase transition between the thermal AdS space and a black hole) was revealed in Schwarzschild-AdS black holes. Subsequently, there has been much interest in studying thermodynamics and phase structure of AdS black holes [9-16]. Interestingly, RN-AdS black holes were found to possess a van der Waals-like phase transition (i.e., a phase transition consisting of a first-order phase transition terminating at a second-order critical point) in a canonical ensemble $[11,12]$ and a Hawking-Page-like phase transition in a grand canonical ensemble [17].

Later, the cosmological constant in AdS black holes is identified as a thermodynamic pressure $[18,19]$. In this framework, the first law becomes consistent with the corresponding Smarr relation, and black hole mass is interpreted as a chemical enthalpy [20]. The phase behavior and $P-V$ criticality have been explored for various AdS black holes in the extended phase space [21-34], which discovered a broad range of new phenomena. For a recent review, see [35]. Specifically for a Schwarzschild-AdS black hole, the coexistence line of 
the Hawking-Page phase transition in the $P-T$ diagram is semi-infinite and reminiscent of the solid/liquid phase transition [36]. In the extended phase space, the analogy between a RN-AdS black hole and the van der Waals fluid becomes more complete, in that the coexistence lines in the $P-T$ diagram are both finite and terminate at critical points, and the $P-V$ criticality matches with one another [19].

In parallel with research on AdS black holes, studies of thermodynamics of black holes in a cavity have also attracted a lot attentions since York realized that Schwarzschild black holes can be thermally stable by placing them inside a spherical cavity, on the wall of which the metric is fixed [37]. Phase structure and transitions of Schwarzschild black holes in a cavity and Schwarzschild-AdS black holes were shown to be strikingly similar [37]. Afterwards, it was found that the phase behavior of RN black holes in a cavity and RN-AdS black holes also has extensive similarities in a grand canonical ensemble [38] and a canonical ensemble $[39,40]$. Similar analysis has been extended to a broad class of brane backgrounds, including $D p$-brane and NS5-brane configurations, and most of the brane systems were observed to undergo Hawking-Page-like or van der Waals-like phase transitions [41-46]. In addition, properties of boson stars and hairy black holes in a cavity were investigated [47-54], which were shown to closely resemble those of holographic superconductors in the AdS gravity. Lately, it was discovered that Gauss-Bonnet black holes in a cavity have quite similar phase structure and transitions to the AdS counterparts [55]. These observations lend support to the analogy between black holes in a cavity and AdS black holes.

However, we recently studied Born-Infeld black holes enclosed in a cavity in a canonical ensemble [56] and a grand canonical ensemble [57] and revealed that their phase structure has dissimilarities from that of Born-Infeld-AdS black holes. Moreover, contrary to the phase behavior, we found that there exist significant differences between the thermodynamic geometry of RN black holes in a cavity and that of RN-AdS black holes [58], and some dissimilarities between the two cases also occur for validities of the second thermodynamic law and the weak cosmic censorship [59]. These findings motivate us to further explore connections between thermodynamic properties of black holes and their boundary conditions. Additionally, it has been proposed [60] that the holographic dual of $T \bar{T}$ deformed $\mathrm{CFT}_{2}$ is a finite region of $\mathrm{AdS}_{3}$ with the wall at finite radial distance, which makes studying properties of black holes in a cavity more attractive. Note that thermodynamics and critical behavior of de Sitter black holes in a cavity were recently investigated in [61-63].

In the existing research on thermodynamic properties of asymptotically flat black holes surrounded by a cavity, thermodynamic quantities that can be interpreted as a pressure or volume are absent. To make analogies with the AdS counterparts and corresponding real-world systems more complete, it is highly desirable to introduce a thermodynamic pressure or volume for black holes in a cavity. To this end, we extend the phase space to include the cavity radius as a new thermodynamic variable, from which the thermodynamic pressure and volume of black holes in a cavity can be established. Particularly in this paper, we confine Schwarzschild and RN black holes in a cavity and study their thermodynamic behavior in the aforementioned extended phase space. In hindsight, the extended phase behavior of Schwarzschild and RN black holes in a cavity is discovered to bear a striking resemblance to that of the AdS counterparts. 
The rest of this paper is organized as follows. Section 2 discusses phase structure of Schwarzschild black holes in a cavity in the extended phase space. In section 3, we investigate the extended phase properties of $\mathrm{RN}$ black holes in a cavity in a canonical ensemble that maintains constant temperature, pressure and charge. In section 4, the Smarr relation for RN black holes in a cavity is derived via Euler's formula and geometric methods. We summarize our results with a brief discussion in section 5 . For simplicity, we set $G=\hbar=c=k_{B}=1$ in this paper.

\section{Phase structure of a Schwarzschild black hole in a cavity}

In this section, we consider a thermodynamic system with a Schwarzschild black hole enclosed in a cavity. The 4-dimensional Schwarzschild black hole solution is described by

$$
d s^{2}=-f(r) d t^{2}+\frac{d r^{2}}{f(r)}+r^{2}\left(d \theta^{2}+\sin ^{2} \theta d \phi^{2}\right), f(r)=1-\frac{r_{+}}{r},
$$

where $r_{+}$is the radius of event horizon. The Hawking temperature $T_{b}$ of the Schwarzschild black hole is

$$
T_{b}=\frac{1}{4 \pi r_{+}} .
$$

Suppose the wall of the cavity enclosing the black hole is at $r=r_{B} \geq r_{+}$and maintained at a temperature of $T$. In [38], it showed that $T$ can be related to $T_{b}$ as

$$
T=\frac{T_{b}}{\sqrt{f\left(r_{B}\right)}} .
$$

The thermal energy $E$ and the Helmholtz free energy $F$ were derived in [39]:

$$
\begin{aligned}
& E=r_{B}\left[1-\sqrt{f\left(r_{B}\right)}\right] \\
& F=r_{B}\left[1-\sqrt{f\left(r_{B}\right)}\right]-T \pi r_{+} .
\end{aligned}
$$

We now introduce a new thermodynamic variable, the thermodynamic volume of the system $V$,

$$
V \equiv \frac{4}{3} \pi r_{B}^{3}
$$

which naturally gives the conjugate thermodynamic pressure,

$$
P=-\frac{\partial E}{\partial V}=-\frac{1}{4 \pi r_{B}^{2}}\left(1-\sqrt{1-x}-\frac{x}{2 \sqrt{1-x}}\right), 0 \leq x \equiv \frac{r_{+}}{r_{B}} \leq 1 .
$$

Extending the phase space of black hole thermodynamics to include the pressure/volume conjugate pair leads to the first law of thermodynamics,

$$
d E=T d S-P d V
$$

where $S=\pi r_{+}^{2}$ is the entropy of black hole, and we use $T=\partial E / \partial S$. If the system undergoes an isobaric process, the Gibbs free energy $G \equiv F+P V$ is employed in the study of phases and phase transitions. 

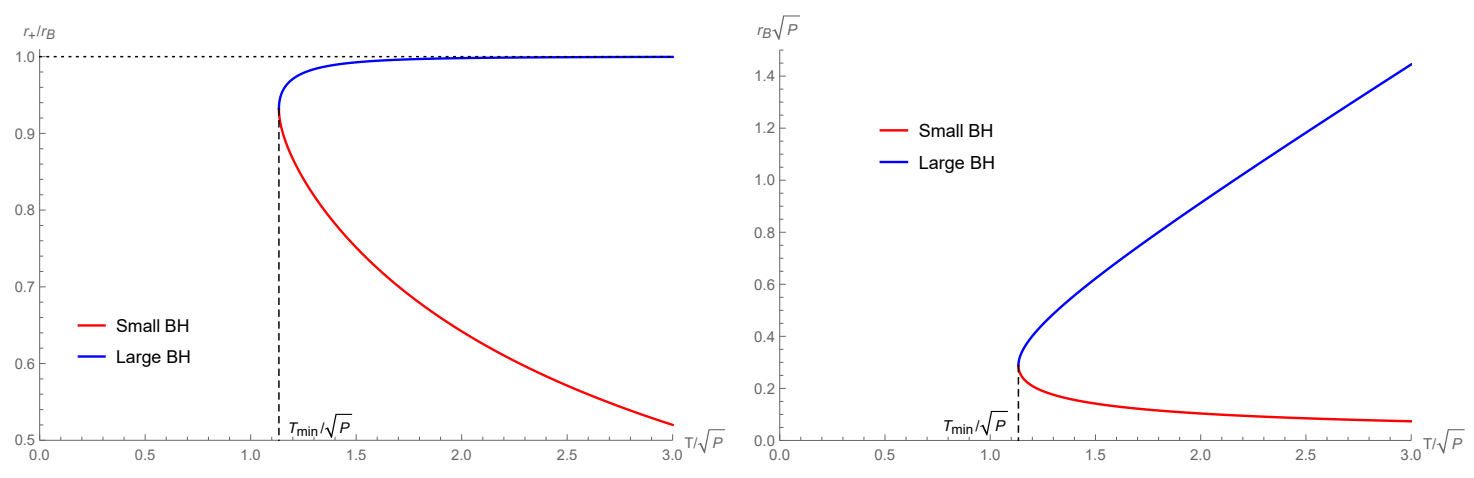

Figure 1. Plots of $r_{+} / r_{B}$ and $r_{B} \sqrt{P}$ against $T / \sqrt{P}$ for a Schwarzschild black hole in a cavity. The red and blue lines represent Small BH and Large BH, respectively. Black hole solutions do not exist when $T<T_{\min }$.

By dimensional analysis, we find that the thermodynamic quantities scale as powers of the pressure $P$,

$$
T=\tilde{T} \sqrt{P}, G=\tilde{G} / \sqrt{P}, r_{B}=\tilde{r}_{B} / \sqrt{P},
$$

where the tildes denote dimensionless quantities. Solving eq. (2.3) for $x$ in terms of $\tilde{T}$ gives $x=x(\tilde{T})$, which is plotted in the left panel of figure 1 . It shows that for $T \geq T_{\min }$, $x=x(\tilde{T})$ is multivalued and consists of two branches, namely Small BH and Large BH. When $T<T_{\min }$, no black hole exists. Plugging $x(\tilde{T})$ into eq. (2.6), one can express $\tilde{r}_{B}$ in terms of $\tilde{T}: \tilde{r}_{B}=\tilde{r}_{B}(\tilde{T})$. In the right panel of figure 1 , we show that $\tilde{r}_{B}(\tilde{T})$ is also composed of the Small BH and Large BH branches. Note that the Small (Large) BH branch possesses smaller (larger) $r_{+}$and $r_{B}$.

To study the thermodynamic stability of the two branches against thermal fluctuations in an isobaric process, we consider the heat capacity at constant pressure,

$$
C_{P}=\tilde{C}_{P} / P=T\left(\frac{\partial S}{\partial T}\right)_{P} .
$$

Using $x(\tilde{T})$, we can rewrite $\tilde{C}_{P}$ as

$$
\tilde{C}_{P}=\tilde{C}_{P}(\tilde{T})
$$

which is presented in the left panel of figure 2. It shows that Small (Large) BH is thermally unstable (stable). To study phase transitions, we also need to consider the thermal flat spacetime as a phase of the system since it is a classical solution in the canonical ensemble. The Gibbs free energy $\tilde{G}(\tilde{T})$ of the two branches and the thermal flat spacetime is displayed in the right panel of figure 2. One finds that the thermal spacetime is the only phase when $\tilde{T}<\tilde{T}_{\text {min }}$, and the black hole appears when $\tilde{T} \geq \tilde{T}_{\text {min }}$. Similar to a Schwarzschild-AdS black hole, a first-order Hawking-Page-like phase transition between the thermal spacetime and Large BH occurs at $\tilde{T}=\tilde{T}_{p}$, where these two phases are of equal Gibbs free energy. The thermal spacetime is globally stable for $\tilde{T}<\tilde{T}_{p}$ while Large BH is globally preferred for $\tilde{T} \geq \tilde{T}_{p}$. The coexistence line of thermal spacetime/Large BH phases is determined by 

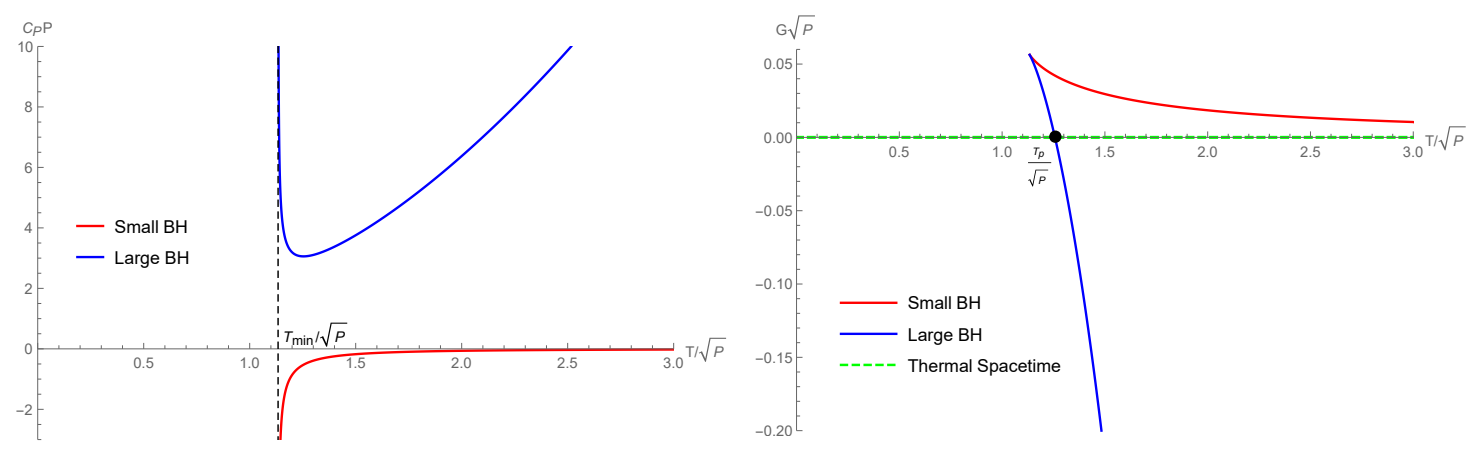

Figure 2. Plots of $C_{p} P$ and $G \sqrt{P}$ against $T / \sqrt{P}$ for a Schwarzschild black hole in a cavity. Left Panel: The heat capacity at constant pressure $C_{p}$ of Small/Large BH is negative/positive, which means that Small/Large BH is thermally unstable/stable in an isobaric process. Right Panel: As $T / \sqrt{P}$ increases from zero, a first-order phase transition from the thermal spacetime to Large BH occurs at the black dot. Above $T_{p} / \sqrt{P}$, Large $\mathrm{BH}$ is the thermodynamically preferred state.

$\tilde{G}=0$, which reads

$$
\left.T\right|_{\text {coexistence }} \simeq 1.257 \sqrt{P} .
$$

The coexistence line is of infinite length and hence reminiscent of the solid/liquid phase transition. It is noteworthy that the coexistence line of thermal AdS spacetime/Large Schwarzschild-AdS BH was given by [35]

$$
\left.T\right|_{\text {coexistence }} \simeq 0.921 \sqrt{P} .
$$

Finally, we discuss the equation of state for the Schwarzschild black hole in a cavity. By rewriting the pressure equation (2.6)whilst using the temperature equation (2.3), we can obtain the equation of state in terms of $V T^{3}$ and $P T^{-2}$, which is depicted as solid lines in figure 3. With fixed $P T^{-2}$, the equation of state has two branches, corresponding to Small BH and Large BH. In the limit of large $V T^{3}$, the equation of state becomes

$$
\begin{aligned}
& V T^{3} \simeq \frac{1}{48 \pi^{2}}\left(\frac{\pi}{2 P T^{-2}}\right)^{3 / 4} \text { for Small } \mathrm{BH}, \\
& V T^{3} \simeq \frac{1}{48 \pi^{2}}\left(\frac{2 \pi}{P T^{-2}}\right)^{3} \quad \text { for Large } \mathrm{BH} .
\end{aligned}
$$

Defining a specific volume $v \equiv(6 V / \pi)^{1 / 3}$, the Large BH equation of state in eq. (2.13) gives the ideal gas law, $P v \simeq T$. We also plot the equation of state for a Schwarzschild-AdS black hole, which is represented by dashed lines in figure 3. Similarly, there exist two branches (i.e., Small $\mathrm{BH}$ and Large $\mathrm{BH}$ ) for a fixed value of $P T^{-2}$. However, only Large $\mathrm{BH}$ exists when $V T^{3}$ is large enough. It is worth noting that, in the limit of large $V T^{3}$, the equation of state for the Schwarzschild-AdS black hole reduces to [35]

$$
V T^{3} \simeq \frac{1}{48 \pi^{2}}\left(\frac{2 \pi}{P T^{-2}}\right)^{3}
$$

which is the same as that for the Large BH branch of the Schwarzschild black hole in a cavity. 


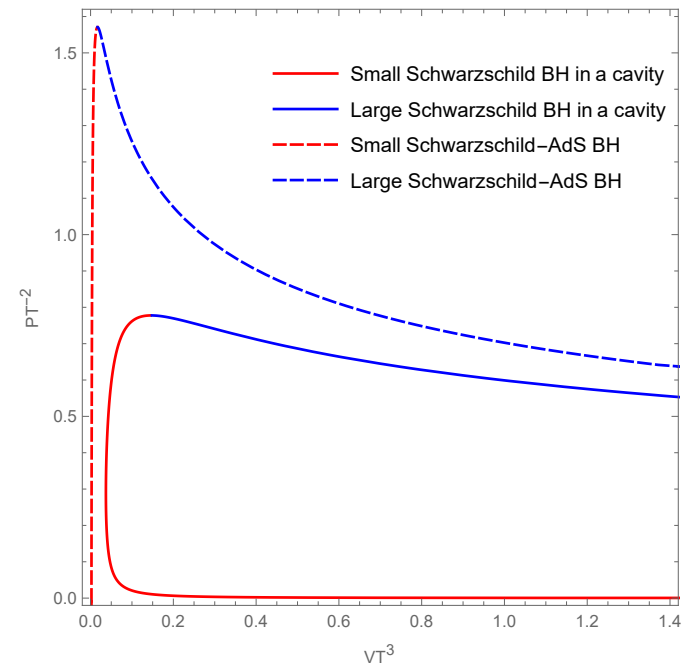

Figure 3. Plot of the equations of state for a Schwarzschild black hole in a cavity and a Schwarzschild-AdS black hole.

\section{Phase structure of a RN black hole in a cavity}

In this section, we discuss phase structure and transitions of a RN black hole in a cavity in the extended phase space. The 4-dimensional RN black hole solution is

$$
\begin{aligned}
d s^{2} & =-f(r) d t^{2}+\frac{d r^{2}}{f(r)}+r^{2}\left(d \theta^{2}+\sin ^{2} \theta d \phi^{2}\right) \\
f(r) & =\left(1-\frac{r_{+}}{r}\right)\left(1-\frac{Q_{b}^{2}}{r_{+} r}\right), A=A_{t}(r) d t=-\frac{Q_{b}}{r} d t
\end{aligned}
$$

where $Q_{b}$ is the black hole charge, and $r_{+}$is the radius of the outer event horizon. The Hawking temperature $T_{b}$ of the RN black hole is given by

$$
T_{b}=\frac{1}{4 \pi r_{+}}\left(1-\frac{Q_{b}^{2}}{r_{+}^{2}}\right) .
$$

In a canonical ensemble, the wall of the cavity, which is located at $r=r_{B}$, is maintained at a temperature of $T$ and a charge of $Q$. It was showed in [38] that the system temperature $T$ and charge $Q$ can be related to the black hole temperature $T_{b}$ and charge $Q_{b}$ as

$$
Q=Q_{b} \text { and } T=\frac{T_{b}}{\sqrt{f\left(r_{B}\right)}}
$$

respectively. For this system, the Helmholtz free energy $F$ and the thermal energy $E$ were also given in [39]

$$
\begin{aligned}
& F=r_{B}\left[1-\sqrt{f\left(r_{B}\right)}\right]-\pi T r_{+}^{2}, \\
& E=r_{B}\left[1-\sqrt{f\left(r_{B}\right)}\right]
\end{aligned}
$$


The physical range of $r_{+}$is constrained by

$$
\frac{r_{e}}{r_{B}} \leq x \equiv \frac{r_{+}}{r_{B}} \leq 1
$$

where $r_{e}=Q$ is the horizon radius of the extremal black hole.

By introducing $V \equiv 4 \pi r_{B}^{3} / 3$ as a thermodynamic variable, the pressure of the system is

$$
P=-\frac{\partial E}{\partial V}=\frac{2 r_{+} r_{B}-Q^{2}-r_{+}^{2}}{8 \pi r_{B}^{3} r_{+} \sqrt{\left(1-\frac{Q^{2}}{r_{+} r_{B}}\right)\left(1-\frac{r_{+}}{r_{B}}\right)}}-\frac{1}{4 \pi r_{B}^{2}} .
$$

In this extend phase space, the first law of thermodynamics is then given by

$$
d E=T d S-P d V+\Phi d Q
$$

where the system's potential $\Phi$ is defined as

$$
\Phi \equiv \frac{A_{t}\left(r_{B}\right)-A_{t}\left(r_{+}\right)}{\sqrt{f\left(r_{B}\right)}}
$$

and we use $d\left[r_{B} f\left(r_{B}\right)\right] / d r_{+}=-4 \pi r_{+} T_{b}$ and $d\left[r_{B} f\left(r_{B}\right)\right] / d Q=-2 \sqrt{f\left(r_{B}\right)} \Phi$. A system under constant pressure in the canonical ensemble is best described by the Gibbs free energy, $G=F+P V$. Two phases in equilibrium have equal Gibbs free energy. Moreover, the heat capacity at constant pressure $C_{P}=T(\partial S / \partial T)_{P}$ is a thermodynamic quantity measuring the stability of a phase in an isobaric process. For later convenience, we can express the thermodynamic quantities in units of $\sqrt{P}$

$$
\tilde{Q} \equiv Q \sqrt{P}, \tilde{r}_{B} \equiv r_{B} \sqrt{P}, \tilde{T} \equiv T / \sqrt{P}, \tilde{G} \equiv G \sqrt{P}, \tilde{C}_{P} \equiv C_{P} P .
$$

Our strategy to discuss the system's phase structure and transitions under constant pressure, temperature and charge will thus be the following: we start from eqs. (3.3) and (3.6) to express $\tilde{T}$ as a function of $\tilde{r}_{B}$ and $\tilde{Q}, \tilde{T}=\tilde{T}\left(\tilde{r}_{B}, \tilde{Q}\right)$. Similarly, using eq. (3.6), one can rewrite the Gibbs free energy and heat capacity as $\tilde{G}=\tilde{G}\left(\tilde{r}_{B}, \tilde{Q}\right)$ and $\tilde{C}_{P}=$ $\tilde{C}_{P}\left(\tilde{r}_{B}, \tilde{Q}\right)$, respectively. After $\tilde{T}=\tilde{T}\left(\tilde{r}_{B}, \tilde{Q}\right)$ is solved for $\tilde{r}_{B}$ in terms of $\tilde{T}$ and $\tilde{Q}$, we can express $\tilde{G}$ and $\tilde{C}_{P}$ with respect to $\tilde{T}$ and $\tilde{Q}, \tilde{G}=\tilde{G}(\tilde{T}, \tilde{Q})$ and $\tilde{C}_{P}=\tilde{C} \tilde{C}_{P}(\tilde{T}, \tilde{Q})$. A critical point occurs at the inflection point of $\tilde{T}$ as a function of $\tilde{r}_{B}$, where

$$
\frac{\partial \tilde{T}}{\partial \tilde{r}_{B}}=0 \text { and } \frac{\partial^{2} \tilde{T}}{\partial \tilde{r}_{B}^{2}}=0
$$

Solving the above equations gives quantities evaluated at the critical point

$$
\left(\tilde{r}_{B c}, \tilde{Q}_{c}, \tilde{T}_{c}, x_{c}\right) \simeq(0.222,0.107,1.100,0.911) .
$$

The critical ratio, $P_{c} v_{c} / T_{c} \simeq 0.405$, where $v \equiv 2 r_{B}$ is the specific volume. Note that $P_{c} v_{c} / T_{c}=3 / 8$ for the van der Waals fluid and a RN-AdS black hole.

With fixed $\tilde{T}$ and $\tilde{Q}$, the number of the phases, corresponding to the branches of $\tilde{r}_{B}(\tilde{T}, \tilde{Q})$, depends on the value of $\tilde{Q}$. When $\tilde{Q}<\tilde{Q}_{c}$, three phases, namely Small BH, 

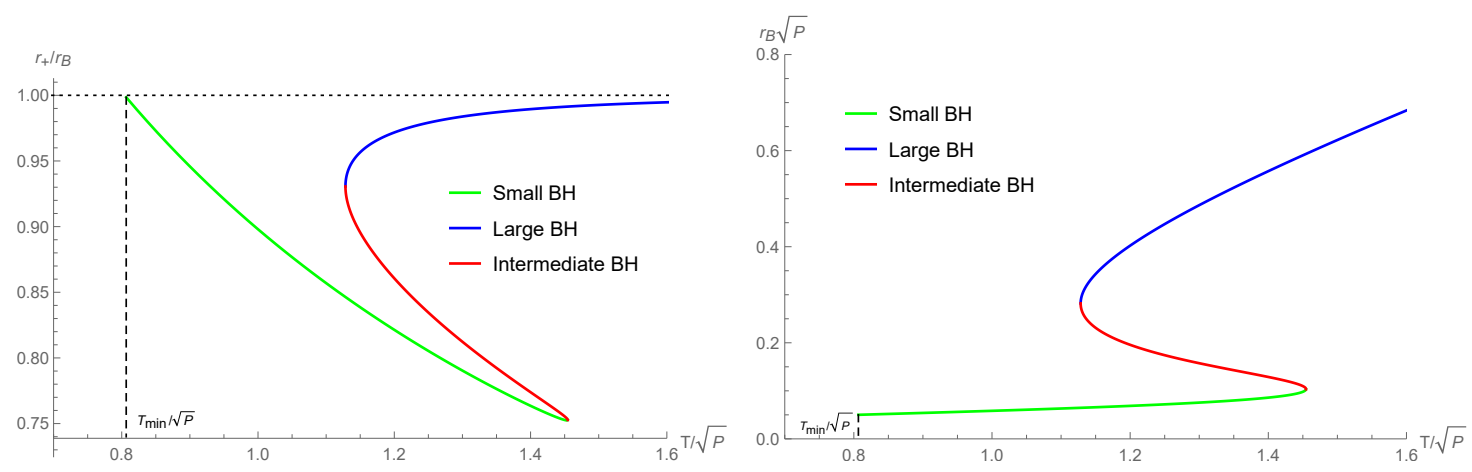

Figure 4. Plots of $r_{h} / r_{B}$ and $r_{B} \sqrt{P}$ against $T / \sqrt{P}$ for a RN black hole in a cavity with $Q=0.05 / \sqrt{P}<Q_{c}$, in which three phases can coexist. The green, red and blue lines represent Small BH, Intermediate BH and Large BH. Black hole solutions do not exist when $T<T_{\min }$.
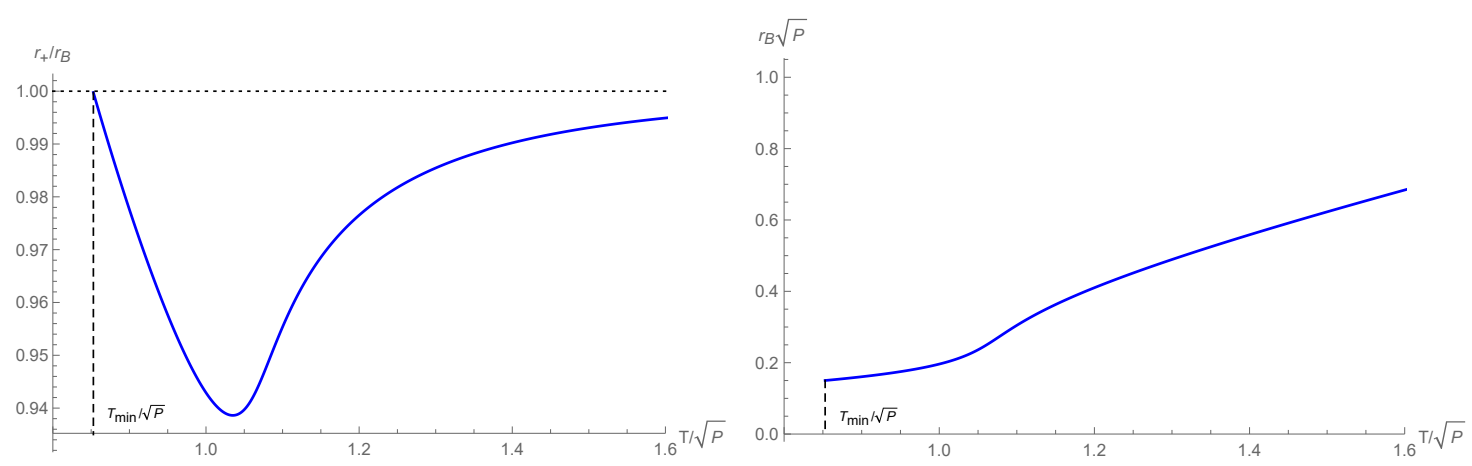

Figure 5. Plots of $r_{h} / r_{B}$ and $r_{B} \sqrt{P}$ against $T / \sqrt{P}$ for a RN black hole in a cavity with $Q=0.15 / \sqrt{P}>Q_{c}$. There is only one phase, which does not exist when $T<T_{\min }$.

Intermediate $\mathrm{BH}$ and Large $\mathrm{BH}$, coexist for some range of $T$. For $\tilde{Q}=0.05<\tilde{Q}_{c}$, we plot $x$ and $\tilde{r}_{B}$ against $\tilde{T}$ in figure 4 , where different colored lines represent different phases. The first thing to note is that there exists a nonzero minimum $\tilde{T}_{\min }$ for Small BH, which means that, unlike a RN-AdS black hole, a RN black hole in a cavity can not become extremal if the pressure is not zero. In other words, eq. (3.6) gives that an extremal RN black hole with $r_{+}=Q$ always has $P=0$. At $\tilde{T}=\tilde{T}_{\min }$, it shows that $x=1$, which corresponds to the black hole with the horizon merging with the wall of the cavity. As $\tilde{T}$ increases, the horizon radius to cavity radius ratio increases toward 1 for Large $\mathrm{BH}$ while decreases for Small BH and Intermediate BH. The cavity enclosing Small BH and Large BH (Intermediate BH) expands (contracts) when $\tilde{T}$ increases. Although it is not shown in the paper, we find that the horizon radius has quite similar behavior to the cavity radius. In figure 5 , we display $x$ and $\tilde{r}_{B}$ against $\tilde{T}$ with $\tilde{Q}=0.15>\tilde{Q}_{c}$, for which there exists only one phase. As one increases $\tilde{T}$ from $\tilde{T}_{\text {min }}$, the size of cavity keeps growing. Meanwhile, the horizon radius to cavity radius ratio first decreases from 1 and then increases towards 1 .

With $\tilde{r}_{B}(\tilde{T}, \tilde{Q})$, we can obtain the heat capacity $\tilde{C}_{P}(\tilde{T}, \tilde{Q})$ and the Gibbs free energy $\tilde{G}(\tilde{T}, \tilde{Q})$ to discuss the stability of phases and phase transitions. Figure 6 shows $\tilde{C}_{P}(\tilde{T}, \tilde{Q})$ and $\tilde{G}(\tilde{T}, \tilde{Q})$ in the left and right panels, respectively, for a RN black hole in a cavity with 

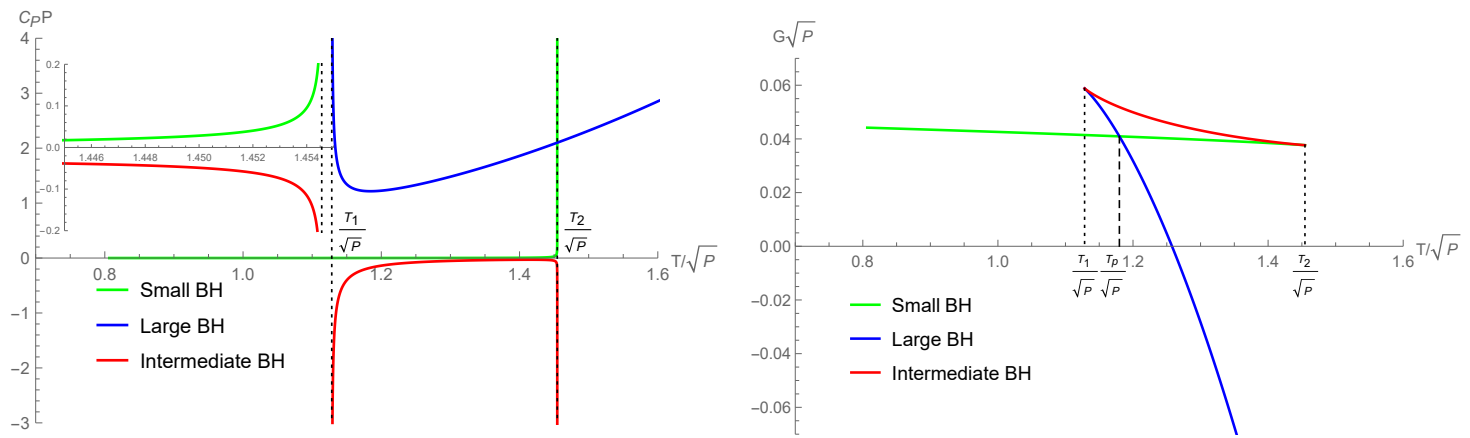

Figure 6. Plots of $C_{p} P$ and $G \sqrt{P}$ against $T / \sqrt{P}$ for a RN black hole in a cavity with $Q=$ $0.05 / \sqrt{P}<Q_{c}$. Left Panel: positive $C_{p}$ means that Large BH and Small BH are thermally stable while negative $C_{p}$ gives Intermediate $\mathrm{BH}$ is thermally unstable. Right Panel: as $T / \sqrt{P}$ increases from $T_{\min } / \sqrt{P}$, a first-order phase transition from Small BH to Large BH occurs at $T=T_{p}$.

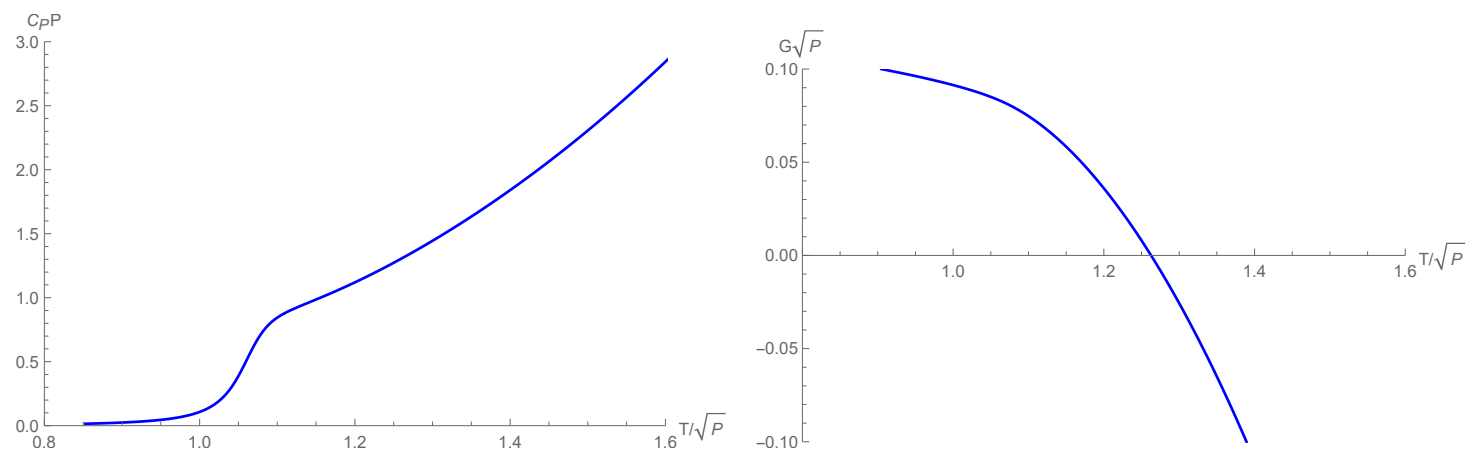

Figure 7. Plots of $C_{p} P$ and $G \sqrt{P}$ against $T / \sqrt{P}$ for a RN black hole in a cavity with $Q=$ $0.15 / \sqrt{P}>Q_{c}$. Left Panel: the only phase has positive heat capacity and hence is thermally stable. Right Panel: there is no phase transition.

$\tilde{Q}=0.05<\tilde{Q}_{c}$. The heat capacity of Small BH and Large BH is positive, which means that Small BH and Large BH are thermally stable. On the other hand, Intermediate BH is a thermally unstable phase. Large BH, Small BH and Intermediate BH coexist when $\tilde{T}_{1}<\tilde{T}<\tilde{T}_{2}$, and there is a first-order phase transition between Small BH and Large BH occurring at $\tilde{T}=\tilde{T}_{p}$ with $\tilde{T}_{1}<\tilde{T}_{p}<\tilde{T}_{2}$. The globally stable phase is Large BH for $T>T_{p}$ and Small BH for $T<T_{p}$. For the system with $\tilde{Q}=0.15>\tilde{Q}_{c}$, there is only one phase, which is always thermally stable (see figure 7 ).

The left and right panels of figure 8 display the globally stable phase of a RN black hole in a cavity in the $P Q^{2}-T Q$ and $\tilde{Q}-\tilde{T}$ planes, respectively. Blue lines represent Large $\mathrm{BH} / \mathrm{Small} \mathrm{BH}$ first-order transition lines. These coexistence lines terminate at the critical point, where a second-order phase transition occurs. These phase diagrams show that the Large $\mathrm{BH} /$ Small $\mathrm{BH}$ phase transition is analogous to the liquid/gas phase transition of the van der Waals fluid. Light red regions mark no BH regions, where $T<T_{\min }$ and hence no black hole solutions exist. Except the existence of no BH regions, these phase diagrams in the cavity case are rather similar to those in the AdS case. 

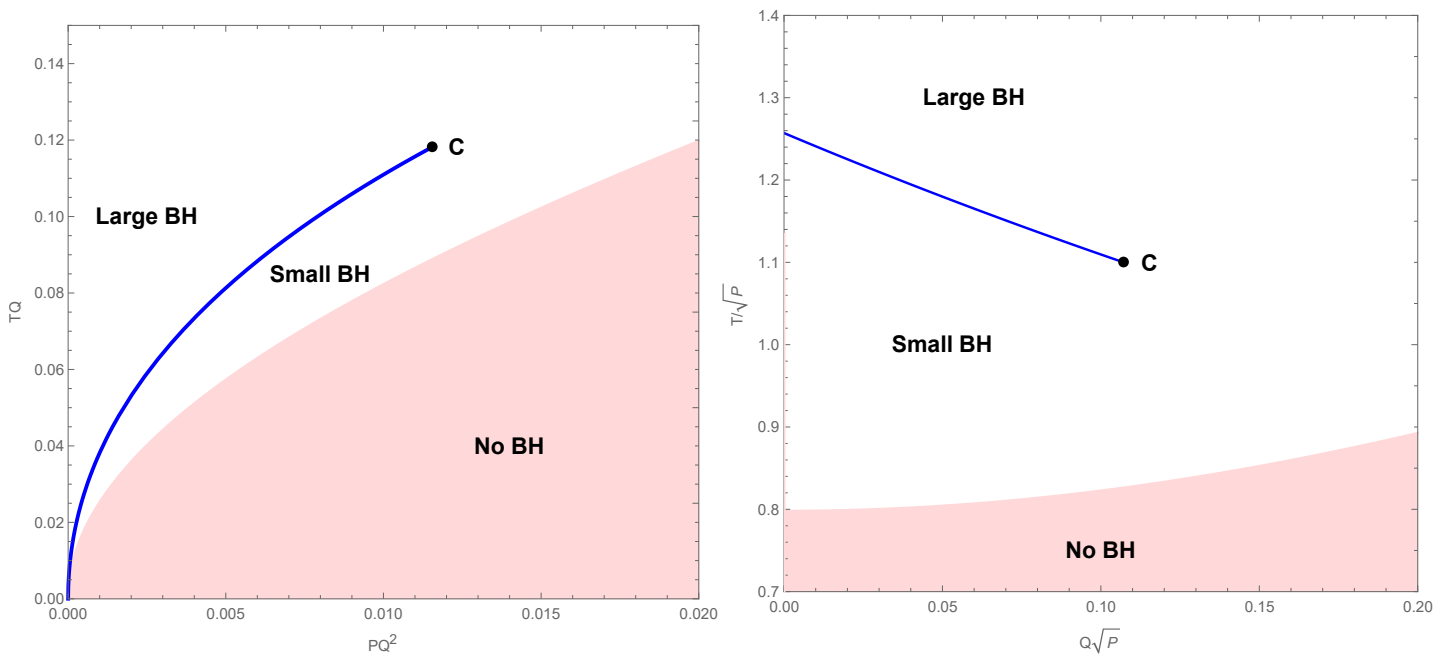

Figure 8. Phase diagrams of a RN-AdS black hole in a cavity in the $P Q^{2}-T Q$ (Left Panel) and $Q \sqrt{P}-T / \sqrt{P}$ (Right Panel) planes. The first-order phase transition lines separating Large $\mathrm{BH}$ and Small BH are displayed by blue lines and terminate at the critical point, marked by black dots. The coexistence lines are of finite length and reminiscent of the liquid/gas phase transition. No BH regions correspond to $T<T_{\min }$, and hence no black hole solutions exist in light red regions.

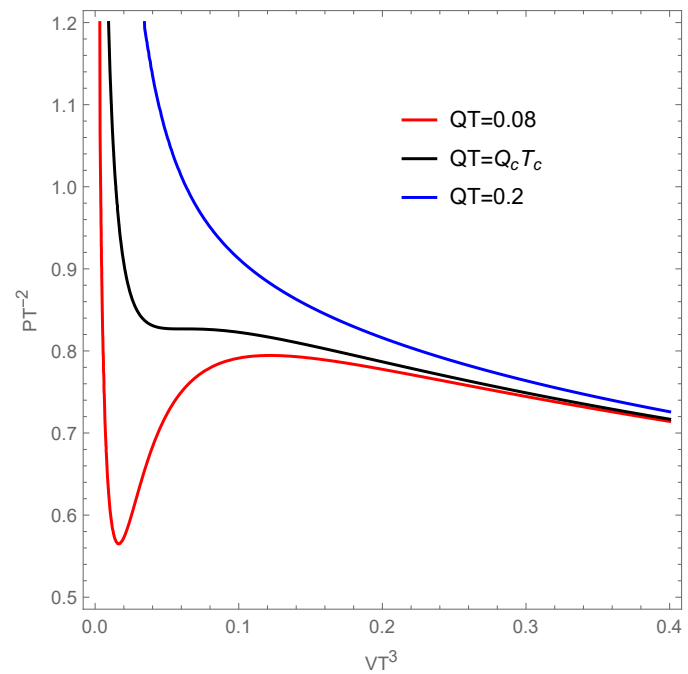

Figure 9. The equations of state for a RN black hole in a cavity for different values of $Q T$. The isotherms with fixed $Q$ bear a striking resemblance to those of the Van der Waals fluid. The oscillating behavior below $Q_{c} T_{c}$ is reminiscent of the liquid/gas phase transition of the Van der Waals fluid.

Finally, we show further analogies with the van der Waals fluid in figure 9 , where the equations of state with $Q T$ ranging from below (red) to above (blue) $Q_{c} T_{c}\left(=\tilde{Q}_{c} \tilde{T}_{c} \simeq 0.118\right.$, black) for a $\mathrm{RN}$ black in a cavity are plotted in the $V T^{3}-P T^{2}$ plane. It is observed that these isotherms in the $V T^{3}-P T^{2}$ plane are strikingly similar to those of van der Waals fluid. In fact, for a RN black in a cavity with $T<\tilde{Q}_{c} \tilde{T}_{c} / Q$, the isotherm in the $V T^{3}-P T^{2}$ 
plane exhibits the oscillating part, in which a fixed $P$ corresponds to three $V$ solutions, namely Small BH, Intermediate BH and Large BH. Such oscillation is reminiscent of the liquid/gas phase transition. Note that the Maxwell's equal area law can determine the Large $\mathrm{BH} / \mathrm{Small} \mathrm{BH}$ phase transition by defining a certain isobar to eliminate the oscillating part, such that the areas above and below the isobar are equal. When $T>\tilde{Q}_{c} \tilde{T}_{c} / Q$, the oscillating behavior of the isotherm disappears, which is reminiscent of the ideal gas law.

\section{Smarr relation}

For an asymptotically flat Kerr-Newman black hole, Smarr [64] showed that the black hole mass $M$ can be written as

$$
M=2 T S+\Phi Q+2 \Omega J
$$

where $T$ represents the Hawking temperature of the black hole, $S$ the entropy, $\Phi$ the electric potential, $Q$ the charge, $\Omega$ the angular velocity, and $J$ the angular momentum. The Smarr relation (4.1) can be derived from the first law of black hole mechanics using Euler's formula for homogeneous functions. In fact, the first law of the asymptotically flat Kerr-Newman black hole reads

$$
d M=T d S+\Phi d Q+\Omega d J
$$

where the ADM mass of the black hole can be identified as a gravitational version of internal energy. Since $M, Q \propto L$, and $J, S \propto L^{2}$, where $L$ is a length scale, we can use Euler's theorem to write $M=M(S, Q, J)$ as

$$
M=2 \frac{\partial M}{\partial S} S+\frac{\partial M}{\partial Q} Q+2 \frac{\partial M}{\partial J} J=2 T S+\Phi Q+2 \Omega J,
$$

where the various partial derivatives are obtained from the first law (4.2).

Similarly, for a four-dimensional AdS black hole in the extended thermodynamics, where the negative cosmological constant is treated as a thermodynamical pressure $P \propto$ $L^{-2}$, the black hole mass $M=M(S, P, Q \cdots)$ is interpreted as a gravitational version of chemical enthalpy [20], and the generalized first law of black hole thermodynamics becomes

$$
d M=T d S+V d P+\Phi d Q+\cdots,
$$

where $V$ is the thermodynamical volume. The Smarr relation then receives an additional $P V$ term,

$$
M=2 \frac{\partial M}{\partial S} S-2 \frac{\partial M}{\partial P} P+\frac{\partial M}{\partial Q} Q+\cdots=2 T S-2 P V+\Phi Q+\cdots,
$$

which can also be derived by geometric means [20].

Here, we use Euler's formula and geometric methods to derive the Smarr relation for a RN black hole in a cavity, which connects the internal energy $E$ or enthalpy $H \equiv E+P V$ of the system with other thermodynamical parameters. Due to Euler's theorem, we can simply write down the internal energy $E=E(S, Q, V)$ as

$$
E=2 \frac{\partial E}{\partial S} S+\frac{\partial E}{\partial Q} Q+3 \frac{\partial E}{\partial V} V
$$


where $V \propto L^{3}$. The first law of the system (3.7) then gives the Smarr relation,

$$
E=2 T S-3 P V+\Phi Q, \text { or } H=2 T S-2 P V+\Phi Q,
$$

which has the same form as the Smarr relation for AdS black holes (4.5). Note that the black hole mass $M$ in eq. (4.5) is treated as the enthalpy.

Alternatively, it is also possible to obtain the Smarr relation using the Komar integral $[6,20]$. In a manifold $\mathcal{M}$ endowed with a time-like Killing vector $K^{\mu}$, we consider a hypersurface $\Sigma$ with the boundary $\partial \Sigma$. Due to Gauss's law and Einstein's equations, integrating the identity for Killing vectors $\nabla_{\mu}\left(\nabla_{\nu} K^{\mu}\right)=K^{\mu} R_{\mu \nu}$ over the hypersurface $\Sigma$ yields

$$
\int_{\partial \Sigma} d S_{\mu \nu} \nabla^{\mu} K^{\nu}=8 \pi \int_{\Sigma} d S_{\mu} K_{\nu}\left(\mathcal{T}^{\mu \nu}-\frac{1}{2} \mathcal{T} g^{\mu \nu}\right)
$$

where $d S_{\mu \nu}$ is the surface element on $\partial \Sigma, d S_{\mu}$ denotes the volume element on $\Sigma$, and $\mathcal{T}^{\mu \nu}$ is the stress-energy tensor. For a RN black hole in a cavity, we choose the hypersurface of constant time $t$ bounded by the Horizon and the cavity radius to be $\Sigma$, such that the boundary $\partial \Sigma$ consists of $r=r_{+}$and $r=r_{B}$. The left-hand side of the identity (4.8) then becomes

$$
\int_{\partial \Sigma} d^{2} x \sqrt{\gamma^{(2)}} \sigma_{\mu} n_{\nu}\left(\nabla^{\mu} K^{\nu}\right)=4 \pi\left(\frac{r_{B}^{2} f^{\prime}\left(r_{B}\right)}{2}-2 T_{b} S\right)=4 \pi \sqrt{f\left(r_{B}\right)}(E+3 P V-2 T S),
$$

where $\sigma_{\mu}=\left(-f^{1 / 2}, 0,0,0\right), n_{\mu}=\left(0, f^{-1 / 2}, 0,0\right), K^{\mu}=(1,0,0,0), \gamma^{(2)}$ is the induced metric on $\partial \Sigma$, and $T_{b}$ is the Hawking temperature. Here, we use eq. (3.4) to express $f^{\prime}\left(r_{B}\right)$ in terms of $\partial E / \partial V(=-P)$. The stress-energy tensor of the electromagnetic field is

$$
\mathcal{T}_{\mu \nu}=F_{\mu \rho} F_{\nu}{ }^{\rho}-\frac{1}{4} g_{\mu \nu} F_{\rho \sigma} F^{\rho \sigma} \text { with } A_{t}(r)=-\frac{Q}{r},
$$

which gives the right-hand side of the identity (4.8),

$$
8 \pi \int_{\Sigma} d^{3} x \sqrt{\gamma^{(3)}} \sigma^{\mu} K^{\nu}\left(\mathcal{T}^{\mu \nu}-\frac{1}{2} \mathcal{T} g^{\mu \nu}\right)=4 \pi Q\left[A_{t}\left(r_{B}\right)-A_{t}\left(r_{+}\right)\right] .
$$

Equating eqs. (4.9) and (4.11), we can obtain the Smarr relation

$$
E=2 T S-3 P V+\Phi Q
$$

where eq. (3.8) is used for $\Phi$.

\section{Discussion and conclusion}

In this paper, we extended the phase space of black holes enclosed in a cavity to include $4 \pi r_{B}^{3} / 3$ as a thermodynamic volume, where $r_{B}$ is the cavity radius. Consequently, the thermodynamic pressure $P$ is define by $P=-\partial E / \partial V$, where $E$ is the thermal energy. Such extension is largely motivated by the extended phase space of AdS black holes, in which the cosmological constant is interpreted as a pressure. We showed that, in these 
extended phase spaces, the thermodynamic behavior of black holes in a cavity closely resembles that of the AdS counterparts, both exhibiting well-known phenomena found in real-world systems.

Specifically, we first investigated phase structure of a Schwarzschild black hole surrounded by a cavity in the extended phase space. We observed two branches of black holes, Large BH and Small BH, above some temperature $T_{\min }$, below which only the thermal flat spacetime exists. The Large BH phase is thermally stable and has larger cavity and horizon radii. Figure 2 revealed that there is a first-order Hawking-Page-like phase transition between the thermal spacetime and Large $\mathrm{BH}$ at $T_{p}>T_{\min }$. Moreover, the coexistence line in the $P-T$ diagram is semi-infinite and hence reminiscent of a solid/liquid phase transition. All these observations indicate that the phase behavior of Schwarzschild black holes in a cavity and Schwarzschild-AdS black holes is quite similar in the extended phase space. However, we noticed in figure 3 that the isotherms of Small BH in the P-V diagram are significantly different in the two cases.

Considering a RN black hole enclosed by a cavity, we demonstrated that the first-order phase transition between Large $\mathrm{BH}$ and Small $\mathrm{BH}$ is similar to the van der Waals phase transition between liquid and gas, in that the Large $\mathrm{BH} / \mathrm{Small} \mathrm{BH}$ coexistence lines in the $T-P$ and $T-Q$ diagrams (see figure 8) are of finite length and end at a second-order critical point. Figure 9 lent further evidence by showing that the isotherms of the RN black hole in a cavity with fixed $Q$ in the $P-V$ diagram behave as those of a van der Waals fluid system. Unlike RN-AdS black holes, RN black holes in a cavity are not admissible in some $(Q, P, T)$-parameter region (e.g., $\mathrm{RN}$ black holes in a cavity with nonzero $P$ can not become extremal). In spite of this difference, the thermodynamic properties of RN black holes in a cavity and RN-AdS black holes are very much alike.

For AdS black holes in the extended thermodynamics as well as ordinary thermodynamic systems, thermodynamic quantities satisfies the (Gibbs-Duhem-)Smarr relation, i.e.,

$$
\text { Enthalpy }=2 \text { Temperature } \times \text { Entropy }-2 \text { Pressure } \times \text { Volume }+\cdots,
$$

which leads to the argument that the cosmological constant must be included as a thermodynamic variable for the thermodynamics of AdS black holes [20]. In this paper, we employed the scaling properties of the thermodynamic quantities and geometric arguments to show that a RN black hole in a cavity also satisfies the Smarr relation (5.1), where the presence of the familiar $P V$ term is crucial for consistency with the Smarr relation. This observation provides a reason to treat the volume (2.5) as a thermodynamic variable for black holes in a cavity.

It showed in [20] that the thermodynamic volume $V$ of a static AdS black hole equals the geometric volume, and can be interpreted as the volume excluded from the spacetime by the black hole horizon. In fact, using the Killing potential, the authors of [20] found that $V$ can be expressed as

$$
V=-\left(V_{\mathrm{BH}}-V_{\mathrm{AdS}}\right) \equiv-\left(\int_{r>r_{+}} d^{D-1} x \sqrt{-g_{\mathrm{BH}}^{(D)}}-\int d^{D-1} x \sqrt{-g_{\mathrm{AdS}}^{(D)}}\right)
$$


where $g_{\mathrm{BH}}^{(D)}$ and $g_{\mathrm{AdS}}^{(D)}$ are the metrics of the $D$-dimensional black hole and AdS space, respectively. Similarly, the thermodynamic volume $V$ of a RN black hole in a cavity can be written as

$$
V=-\left(V_{\text {Cavity }}-V_{\text {Flat }}\right) \equiv-\left(\int_{r>r_{B}} d r d \theta d \phi \sqrt{-g_{\mathrm{RN}}}-\int d r d \theta d \phi \sqrt{-g_{\text {Flat }}}\right),
$$

$g_{\mathrm{RN}}$ and $g_{\text {Flat }}$ are the metrics of the 4-dimensional RN black hole and flat space, respectively. The thermodynamic volume $V$ in the cavity case is identical to the "geometric volume" of a ball of radius $r_{B}$ in flat space, and measures the volume excluded from the spacetime by the cavity.

In existing studies, thermodynamic properties of black holes in a cavity were investigated in the normal phase space, in which the cavity radius is fixed, and found to closely resemble those of the AdS counterparts for various black holes. In this paper, we extended the phase space to include the thermodynamic volume and showed that analogies with the AdS counterparts still hold for Schwarzschild and RN black holes in the extended phase space. On the other hand, AdS black holes have been observed to possess a lot richer phase structure and transitions, e.g., reentrant phase transitions and triple points, in the extended phase space. It would be very interesting to explore these phenomena in the context of black holes in a cavity and check whether analogies with the AdS counterparts can go beyond Schwarzschild and RN black holes.

\section{Acknowledgments}

We are grateful to Shuxuan Ying and Zhipeng Zhang for useful discussions and valuable comments. This work is supported in part by NSFC (Grant No. 11875196, 11375121, 11947225 and 11005016).

Open Access. This article is distributed under the terms of the Creative Commons Attribution License (CC-BY 4.0), which permits any use, distribution and reproduction in any medium, provided the original author(s) and source are credited.

\section{References}

[1] S.W. Hawking, Gravitational radiation from colliding black holes, Phys. Rev. Lett. 26 (1971) 1344 [INSPIRE].

[2] J.D. Bekenstein, Black holes and the second law, Lett. Nuovo Cim. 4 (1972) 737 [InSPIRE].

[3] J.D. Bekenstein, Black holes and entropy, Phys. Rev. D 7 (1973) 2333 [InSPIRE].

[4] S.W. Hawking, Black hole explosions, Nature 248 (1974) 30 [INSPIRE].

[5] S.W. Hawking, Particle Creation by Black Holes, in 1st Oxford Conference on Quantum Gravity, Oxford U.K. (1975), pg. 219.

[6] J.M. Bardeen, B. Carter and S.W. Hawking, The Four laws of black hole mechanics, Commun. Math. Phys. 31 (1973) 161 [INSPIRE]. 
[7] J.M. Maldacena, The Large N limit of superconformal field theories and supergravity, Int. J. Theor. Phys. 38 (1999) 1113 [hep-th/9711200] [INSPIRE].

[8] S.W. Hawking and D.N. Page, Thermodynamics of Black Holes in anti-de Sitter Space, Commun. Math. Phys. 87 (1983) 577 [inSPIRE].

[9] E. Witten, Anti-de Sitter space, thermal phase transition, and confinement in gauge theories, Adv. Theor. Math. Phys. 2 (1998) 505 [hep-th/9803131] [InSPIRE].

[10] M. Cvetič and S.S. Gubser, Phases of R charged black holes, spinning branes and strongly coupled gauge theories, JHEP 04 (1999) 024 [hep-th/9902195] [INSPIRE].

[11] A. Chamblin, R. Emparan, C.V. Johnson and R.C. Myers, Charged AdS black holes and catastrophic holography, Phys. Rev. D 60 (1999) 064018 [hep-th/9902170] [INSPIRE].

[12] A. Chamblin, R. Emparan, C.V. Johnson and R.C. Myers, Holography, thermodynamics and fluctuations of charged AdS black holes, Phys. Rev. D 60 (1999) 104026 [hep-th/9904197] [INSPIRE].

[13] M.M. Caldarelli, G. Cognola and D. Klemm, Thermodynamics of Kerr-Newman-AdS black holes and conformal field theories, Class. Quant. Grav. 17 (2000) 399 [hep-th/9908022] [INSPIRE].

[14] R.-G. Cai, Gauss-Bonnet black holes in AdS spaces, Phys. Rev. D 65 (2002) 084014 [hep-th/0109133] [INSPIRE].

[15] M. Cvetič, S. Nojiri and S.D. Odintsov, Black hole thermodynamics and negative entropy in de Sitter and anti-de Sitter Einstein-Gauss-Bonnet gravity, Nucl. Phys. B 628 (2002) 295 [hep-th/0112045] [INSPIRE].

[16] S. Nojiri and S.D. Odintsov, Anti-de Sitter black hole thermodynamics in higher derivative gravity and new confining deconfining phases in dual CFT, Phys. Lett. B 521 (2001) 87 [Erratum ibid. 542 (2002) 301] [hep-th/0109122] [INSPIRE].

[17] C.S. Peca and J.P.S. Lemos, Thermodynamics of Reissner-Nordstrom anti-de Sitter black holes in the grand canonical ensemble, Phys. Rev. D 59 (1999) 124007 [gr-qc/9805004] [INSPIRE].

[18] B.P. Dolan, Pressure and volume in the first law of black hole thermodynamics, Class. Quant. Grav. 28 (2011) 235017 [arXiv:1106.6260] [INSPIRE].

[19] D. Kubiznak and R.B. Mann, P-V criticality of charged AdS black holes, JHEP 07 (2012) 033 [arXiv: 1205.0559] [INSPIRE].

[20] D. Kastor, S. Ray and J. Traschen, Enthalpy and the Mechanics of AdS Black Holes, Class. Quant. Grav. 26 (2009) 195011 [arXiv:0904.2765] [InSPIRE].

[21] S.-W. Wei and Y.-X. Liu, Critical phenomena and thermodynamic geometry of charged Gauss-Bonnet AdS black holes, Phys. Rev. D 87 (2013) 044014 [arXiv: 1209.1707] [InSPIRE].

[22] S. Gunasekaran, R.B. Mann and D. Kubiznak, Extended phase space thermodynamics for charged and rotating black holes and Born-Infeld vacuum polarization, JHEP 11 (2012) 110 [arXiv:1208.6251] [INSPIRE].

[23] R.-G. Cai, L.-M. Cao, L. Li and R.-Q. Yang, P-V criticality in the extended phase space of Gauss-Bonnet black holes in AdS space, JHEP 09 (2013) 005 [arXiv:1306.6233] [INSPIRE].

[24] W. Xu and L. Zhao, Critical phenomena of static charged AdS black holes in conformal gravity, Phys. Lett. B 736 (2014) 214 [arXiv:1405.7665] [INSPIRE]. 
[25] A.M. Frassino, D. Kubiznak, R.B. Mann and F. Simovic, Multiple Reentrant Phase Transitions and Triple Points in Lovelock Thermodynamics, JHEP 09 (2014) 080 [arXiv: 1406.7015] [INSPIRE].

[26] M.H. Dehghani, S. Kamrani and A. Sheykhi, $P-V$ criticality of charged dilatonic black holes, Phys. Rev. D 90 (2014) 104020 [arXiv: 1505. 02386] [INSPIRE].

[27] R.A. Hennigar, W.G. Brenna and R.B. Mann, $P-v$ criticality in quasitopological gravity, JHEP 07 (2015) 077 [arXiv: 1505.05517] [INSPIRE].

[28] E. Caceres, P.H. Nguyen and J.F. Pedraza, Holographic entanglement entropy and the extended phase structure of STU black holes, JHEP 09 (2015) 184 [arXiv:1507.06069] [INSPIRE].

[29] S.H. Hendi, G.-Q. Li, J.-X. Mo, S. Panahiyan and B. Eslam Panah, New perspective for black hole thermodynamics in Gauss-Bonnet-Born-Infeld massive gravity, Eur. Phys. J. C 76 (2016) 571 [arXiv: 1608.03148] [INSPIRE].

[30] S.H. Hendi, R.B. Mann, S. Panahiyan and B. Eslam Panah, Van der Waals like behavior of topological AdS black holes in massive gravity, Phys. Rev. D 95 (2017) 021501 [arXiv: 1702.00432] [INSPIRE].

[31] J.P.S. Lemos and O.B. Zaslavskii, Black hole thermodynamics with the cosmological constant as independent variable: Bridge between the enthalpy and the Euclidean path integral approaches, Phys. Lett. B 786 (2018) 296 [arXiv:1806.07910] [INSPIRE].

[32] J.F. Pedraza, W. Sybesma and M.R. Visser, Hyperscaling violating black holes with spherical and hyperbolic horizons, Class. Quant. Grav. 36 (2019) 054002 [arXiv:1807.09770] [INSPIRE].

[33] P. Wang, H. Wu and H. Yang, Thermodynamics and Phase Transitions of Nonlinear Electrodynamics Black Holes in an Extended Phase Space, JCAP 04 (2019) 052 [arXiv: 1808.04506] [INSPIRE].

[34] S.-W. Wei and Y.-X. Liu, Extended thermodynamics and microstructures of four-dimensional charged Gauss-Bonnet black hole in AdS space, Phys. Rev. D 101 (2020) 104018 [arXiv:2003.14275] [INSPIRE].

[35] D. Kubiznak, R.B. Mann and M. Teo, Black hole chemistry: thermodynamics with Lambda, Class. Quant. Grav. 34 (2017) 063001 [arXiv:1608.06147] [INSPIRE].

[36] D. Kubiznak and R.B. Mann, Black hole chemistry, Can. J. Phys. 93 (2015) 999 [arXiv: 1404.2126] [INSPIRE].

[37] J.W. York, Black hole thermodynamics and the Euclidean Einstein action, Phys. Rev. D 33 (1986) 2092 [INSPIRE].

[38] H.W. Braden, J. Brown, B.F. Whiting and J.W. York, Charged black hole in a grand canonical ensemble, Phys. Rev. D 42 (1990) 3376 [inSPIRE].

[39] S. Carlip and S. Vaidya, Phase transitions and critical behavior for charged black holes, Class. Quant. Grav. 20 (2003) 3827 [gr-qc/0306054] [INSPIRE].

[40] A.P. Lundgren, Charged black hole in a canonical ensemble, Phys. Rev. D 77 (2008) 044014 [gr-qc/0612119] [INSPIRE].

[41] J.X. Lu, S. Roy and Z. Xiao, Phase transitions and critical behavior of black branes in canonical ensemble, JHEP 01 (2011) 133 [arXiv:1010.2068] [INSPIRE]. 
[42] C. Wu, Z. Xiao and J. Xu, Bubbles and Black Branes in Grand Canonical Ensemble, Phys. Rev. D 85 (2012) 044009 [arXiv: 1108.1347] [InSPIRE].

[43] J.X. Lu, R. Wei and J. Xu, The phase structure of black D1/D5 (F/NS5) system in canonical ensemble, JHEP 12 (2012) 012 [arXiv:1210.0708] [INSPIRE].

[44] J.X. Lu and R. Wei, Modulating the phase structure of black D6 branes in canonical ensemble, JHEP 04 (2013) 100 [arXiv:1301.1780] [INSPIRE].

[45] D. Zhou and Z. Xiao, Phase structures of the black $D p-D(p+4)$-brane system in various ensembles I: thermal stability, JHEP 07 (2015) 134 [arXiv: 1502.00261] [INSPIRE].

[46] Z. Xiao and D. Zhou, Phase structures of the black $D p-D(p+4)$-brane system in various ensembles II: electrical and thermodynamic stability, JHEP 09 (2015) 028 [arXiv: 1507.02088] [INSPIRE].

[47] N. Sanchis-Gual, J.C. Degollado, P.J. Montero, J.A. Font and C. Herdeiro, Explosion and Final State of an Unstable Reissner-Nordström Black Hole, Phys. Rev. Lett. 116 (2016) 141101 [arXiv: 1512.05358] [INSPIRE].

[48] N. Sanchis-Gual, J.C. Degollado, C. Herdeiro, J.A. Font and P.J. Montero, Dynamical formation of a Reissner-Nordström black hole with scalar hair in a cavity, Phys. Rev. D 94 (2016) 044061 [arXiv: 1607.06304] [INSPIRE].

[49] P. Basu, C. Krishnan and P.N. Bala Subramanian, Hairy Black Holes in a Box, JHEP 11 (2016) 041 [arXiv: 1609.01208] [INSPIRE].

[50] Y. Peng, B. Wang and Y. Liu, On the thermodynamics of the black hole and hairy black hole transitions in the asymptotically flat spacetime with a box, Eur. Phys. J. C 78 (2018) 176 [arXiv: 1708.01411] [INSPIRE].

[51] Y. Peng, Studies of a general flat space/boson star transition model in a box through a language similar to holographic superconductors, JHEP 07 (2017) 042 [arXiv: 1705. 08694] [INSPIRE].

[52] Y. Peng, Scalar field configurations supported by charged compact reflecting stars in a curved spacetime, Phys. Lett. B $\mathbf{7 8 0}$ (2018) 144 [arXiv:1801.02495] [INSPIRE].

[53] B. Kiczek and M. Rogatko, Ultra-compact spherically symmetric dark matter charged star objects, JCAP 09 (2019) 049 [arXiv: 1904.07232] [INSPIRE].

[54] B. Kiczek and M. Rogatko, Influence of dark matter on black hole scalar hair, Phys. Rev. D 101 (2020) 084035 [arXiv: 2004.06617] [INSPIRE].

[55] P. Wang, H. Yang and S. Ying, Thermodynamics and phase transition of a Gauss-Bonnet black hole in a cavity, Phys. Rev. D 101 (2020) 064045 [arXiv: 1909.01275] [InSPIRE].

[56] P. Wang, H. Wu and H. Yang, Thermodynamics and Phase Transition of a Nonlinear Electrodynamics Black Hole in a Cavity, JHEP 07 (2019) 002 [arXiv: 1901.06216] [INSPIRE].

[57] K. Liang, P. Wang, H. Wu and M. Yang, Phase structures and transitions of Born-Infeld black holes in a grand canonical ensemble, Eur. Phys. J. C 80 (2020) 187 [arXiv: 1907.00799] [INSPIRE].

[58] P. Wang, H. Wu and H. Yang, Thermodynamic Geometry of AdS Black Holes and Black Holes in a Cavity, Eur. Phys. J. C 80 (2020) 216 [arXiv:1910.07874] [InSPIRE].

[59] P. Wang, H. Wu and S. Ying, Validity of Thermodynamic Laws and Weak Cosmic Censorship for AdS Black Holes and Black Holes in a Cavity, arXiv:2002.12233 [INSPIRE]. 
[60] L. McGough, M. Mezei and H. Verlinde, Moving the CFT into the bulk with TTT, JHEP 04 (2018) 010 [arXiv: 1611.03470] [INSPIRE].

[61] F. Simovic and R. Mann, Critical Phenomena of Charged de Sitter Black Holes in Cavities, Class. Quant. Grav. 36 (2019) 014002 [arXiv:1807.11875] [INSPIRE].

[62] F. Simovic and R.B. Mann, Critical Phenomena of Born-Infeld-de Sitter Black Holes in Cavities, JHEP 05 (2019) 136 [arXiv: 1904.04871] [INSPIRE].

[63] S. Haroon, R.A. Hennigar, R.B. Mann and F. Simovic, Thermodynamics of Gauss-Bonnet-de Sitter Black Holes, Phys. Rev. D 101 (2020) 084051 [arXiv:2002.01567] [INSPIRE].

[64] L. Smarr, Mass formula for Kerr black holes, Phys. Rev. Lett. 30 (1973) 71 [Erratum ibid. 30 (1973) 521] [INSPIRE]. 